\title{
Short-term outcomes of laparoscopic versus open total gastrectomy after neoadjuvant chemotherapy: a cohort study using the propensity score matching method
}

\author{
Yinkui Wang", Xiaokang Lei", Zining Liu\#, Fei Shan, Xiangji Ying, Ziyu Li, Jiafu Ji \\ Key Laboratory of Carcinogenesis and Translational Research (Ministry of Education/Beijing), Gastrointestinal Cancer Center, Peking University \\ Cancer Hospital \& Institute, Beijing, China \\ Contributions: (I) Conception and design: Y Wang, Z Liu, Z Li; (II) Administrative support: Z Li, J Ji; (III) Provision of study materials or patients: \\ Y Wang, Z Liu, X Lei; (IV) Collection and assembly of data: X Lei, Z Liu, Y Wang; (V) Data analysis and interpretation: Z Liu, X Ying; (VI) \\ Manuscript writing: All authors; (VII) Final approval of manuscript: All authors. \\ \#These authors contributed equally to this work. \\ Correspondence to: Ziyu Li, MD, PhD. Key Laboratory of Carcinogenesis and Translational Research (Ministry of Education/Beijing), Gastrointestinal \\ Cancer Center, Peking University Cancer Hospital and Institute, Beijing, China. Email: ziyu_li@hsc.pku.edu.cn.
}

\begin{abstract}
Background: Until now, little is known about the benefit of laparoscopic total gastrectomy (LTG) after neoadjuvant chemotherapy (NACT). This study was designed to compare the safety and efficacy of the LTG versus the open total gastrectomy (OTG) approach after NACT treatment in patients with advanced gastric cancer (AGC).

Methods: This study involved a cohort of 145 patients with AGC who underwent total gastrectomy after NACT at our centre between April 2013 and August 2018 including 24 cases of LTG and 121 OTG. The baseline characteristics were matched based on 1:2 balanced propensity score-matching method.

Results: Sixty-nine marched cases were finally analysed (23 LTG vs. 46 OTG). All patients underwent R0 resection. Compared to the OTG group, the LTG group had a longer surgery duration $(\mathrm{P}<0.001)$, but a shorter incision length $(\mathrm{P}<0.001)$ and less intravenous patient-controlled analgesia $(\mathrm{IV}-\mathrm{PCA})$ time after surgery $(\mathrm{P}=0.027)$. No statistical differences were observed in terms of blood loss, retrieved lymph nodes (LNs), resection margin, length of stay, postoperative pain intensity, and complications $(\mathrm{P}>0.05)$.

Conclusions: LTG had comparable safety and histological findings to OTG after NACT in the perioperative period; however, LTG is less invasive and patients can benefit from less IV-PCA use. Further research is needed to investigate long-term effects.
\end{abstract}

Keywords: Gastric cancer (GC); neoadjuvant chemotherapy (NACT); safety; laparoscopic technique

Submitted Sep 06, 2020. Accepted for publication Jan 11, 2021.

doi: 10.21037/jgo-20-374

View this article at: http://dx.doi.org/10.21037/jgo-20-374

\section{Introduction}

Gastric cancer (GC) is the fifth most commonly diagnosed neoplasm, accounting for $5.7 \%$ of all cancers worldwide (1). In China, GC is the second leading cause of cancer-related death; there is a substantial number of newly discovered cases each year, with more than $80 \%$ of patients in an advanced stage (2-4). There are many reasons for the high incidence of stomach cancer in China including regional factors, the early detection rate, and the ageing population $(3,5)$. Since Azagra et al. first performed total gastrectomy for GC in 1999 (6), laparoscopic total gastrectomy (LTG) has been widely recognised for its surgical feasibility and better postoperative outcomes $(7,8)$. The reported advantages of LTG include better visualisation, less invasive surgery, less postoperative pain, and shorter hospital stay, among others (8-10). 
Although surgery is the only curative approach for patients with GC, most patients with GC are in an advanced stage at diagnosis, which dramatically lowers the $\mathrm{R} 0$ resection rate, leading to poorer prognosis $(11,12)$. Neoadjuvant chemotherapy (NACT) is now regarded as an effective tumour downstaging approach, improving the $\mathrm{R} 0$ resection rate, and thereby, improving long-term survival (13-16). Based on the evidence above, perioperative treatment protocols have been introduced into the national guidelines in many Western countries. Further, clinical stage (cTNM) and post-preoperative therapy stage (ypTNM) have been newly defined in the recent $8^{\text {th }}$ edition of the AJCC Cancer Staging Manual $(17,18)$.

With the wide use of LTG and NACT in patients with advanced gastric cancer (AGC), there is increasing interest in their combined benefit for patients as compared to the effect of a single treatment. In this study, we utilised retrospective cohort data to evaluate the safety and histological findings of NACT followed by LTG treatment in patients with AGC. We present the study in accordance with the STROBE reporting checklist (available at http:// dx.doi.org/10.21037/jgo-20-374).

\section{Methods}

The study was conducted in accordance with the Declaration of Helsinki (as revised in 2013). The study was approved by Ethics Committee of Beijing Cancer Hospital (No. 2019YJZ26) and informed consent was taken from all the patients.

\section{Eligibility}

The study included locally advanced proximal GC patients (cT2-cT4a) who received 2-5 cycles of NACT before surgical treatment. All included patients were patients at our centre between April 2013 and August 2018. The determination of clinical stages, design for treatment route, preoperative assessment, and prompt intervention for adverse events were managed by the multidisciplinary team (MDT). All patients had CT scan record for their baseline evaluation. Started from 2014, the pretherapeutic laparoscopic staging was designed for all patients that required NACT treatment.

To ensure the enough NACT treatment exposure, all included patients received at least two cycles of NACT before surgery; the regimens used included DC (docetaxel and cisplatin), DS (docetaxel and S-1), DX (paclitaxel and capecitabine), EP (cisplatin and etoposide), FOLFIRI (leucovorin calcium, 5-fluorouracil, and irinotecan), POS (paclitaxel and oxaliplatin and S-1), S-1, SEEOX (oxaliplatin, etoposide, epirubicin, and S-1), SOX (oxaliplatin and TS-1), and CapeOX (capecitabine and oxaliplatin) (Table S1). To assess the influence of the number of cycles, the three 14-day cycles of FOLFIRI and POS were calculated as two 21-day cycles and were transformed based on the rounding strategies.

Patients were excluded if they had other malignant diseases in the past five years, underwent upper abdomen surgery other than laparoscopic cholecystectomy, or had medical conditions requiring emergency surgeries.

The surgery approach, either LTG or open total gastrectomy (OTG), was decided based on each patient's preference. If a patient was not sure of the surgical approach (no preference), the chief surgeon would decide which technique to use according to actual circumstances. Generally, a laparoscopic exploration would be done at first to measure the suitability for the two approaches. Overall, 24 patients who underwent LTG and 121 patients who underwent OTG following NACT were available for propensity score matching (PSM).

\section{Surgical technique}

All patients enrolled in our study (both the LTG and OTG groups) underwent standard total gastrectomy with D2 lymphadenectomy [including lymph node (LN) Nos. 1-7, 8a, 9, 10,11p, 11d, and 12a], according to the latest Japanese Gastric Cancer Association treatment guidelines (19). Each LN group was tagged instantly after resection and separately processed for further examination after surgery.

LTG was performed with the five-trocar approach, including one $10 \mathrm{~mm}$ trocar below the umbilicus for the camera, three $10 \mathrm{~mm}$ trocars in the left upper quadrant $2 \mathrm{~cm}$ below the left lower rib margin and in the right and left flank areas, respectively, and one $5 \mathrm{~mm}$ trocar placed in the right upper quadrant $2 \mathrm{~cm}$ below the right lower rib margin. The specimens were pulled out through the extended umbilical port (about 3-4 cm). OTG was performed through a 20 to $25 \mathrm{~cm}$ incision (approximately) from the falciform process to the periumbilical area. In all cases, Roux-Y reconstruction was performed after the gastrectomy during which an oesophagojejunostomy was achieved using a linear (side-to-side anastomosis) or circular stapler. The type of anastomosis depended on the clinical 
situation and surgeon's preference. All procedures were performed by one surgical team with extensive experience in both open and laparoscopic GC surgeries.

During the perioperative period, all patients were prescribed intravenous patient-controlled analgesia (IV-PCA) and were managed according to a standardised clinical pathway. Discharge was recommended when the patient tolerated more than two days of soft diet without abdominal pain or fever.

\section{Data collection and outcome assessment}

Demographic characteristics were collected from all the patients, including age, gender, body mass index (BMI), comorbidities, physical status according to the American Society of Anesthesiology (ASA), and pathological features. Enhanced abdominal computed tomography (CT) was conducted before and after NACT in each patient to assess the NACT response according to the Response Evaluation Criteria in Solid Tumours (RECIST version 1.1) (20). Pathological and clinical staging was reported according to the 8th edition of the International Union Against Cancer (UICC) TNM classification.

Surgery-related indices included the length of incision, surgery time, estimated blood loss, number of retrieved LNs, proximal and distal margin of the tumour, first aerofluxus time, time to first defecation, time to resumption of liquid diet, time to drainage tube removal, and the length of hospital stay. The distance of tumour to the margin was determined to be the closest and farthest points of extension of any discrete mass to the given margin based on gross examination intraoperatively. Visual analogue scale (VAS) (21) was used to evaluate pain intensity at 24,48 , and 72 hours post-surgery. Supplementary opioid use after surgery was carefully recorded in every patient and converted to oral morphine equivalents (OME) (22). The time of IV-PCA use and supplementary morphine consumption in each patient was recorded. Postoperative complications were defined as problems affecting patients during their hospital stay following surgery, including, anastomotic leakage, peritoneal cavity infection, pulmonary infection, haemorrhage etc., graded by the Clavien-Dindo classification system (23). Hospital mortality was defined as death occurring within 30 days of initial surgery, regardless of cause.

\section{PSM}

Examination of the baseline characteristics revealed a significant difference in tumour size between the groups (Table S2). To minimise the impact of latent selective bias, 1:2 matching was performed between the LTG and OTG groups based on the propensity score using a 0.15 caliper width. We used a multivariate logistic regression model to calculate propensity scores for each patient in each group. Selected covariates, including age, BMI, gender, ASA score, tumour diameter, NACT regimen, and cycles, were either the identified confounders or the important baseline factors. Following PSM, a cohort of 23 LTG patients and 46 matched OTG patients was formed. PSM was performed using the R "MatchIt" package (R Version 3.6.2) (24). A detailed patients selection method and the distribution of propensity score before and after PSM are shown in Figures 1 and 2, respectively.

\section{Statistical analysis}

All baseline characteristics (pre-match and post-match) were analyzed using standardized mean difference (SMD), where SMD values $>0.1$ were considered to indicate a statistically significant difference between groups. For outcome variables, quantitative data are presented as mean \pm standard deviation or as median (IQR) if the data were highly skewed. Independent $t$-tests, Wilcoxon rank-sum tests, Chi-square tests, and Fisher's exact tests were used to test for differences in baseline data and outcomes of surgeries between the OTG and LTG groups. P value lesser than 0.05 was considered statistically significant. STATA ver. 15.0 (StataCorp. 2017. Stata Statistical Software: Release 15. College Station, TX: StataCorp LLC.) was used for all statistical analyses.

\section{Results}

\section{Demographic and clinicopathologic characteristics}

After application of the inclusion and exclusion criteria, 145 patients were enrolled in this study ( 24 cases who underwent LTG and 121 cases who underwent OTG). The two groups differed significantly in tumor diameter (long axis: $3.33 \pm 1.36$ vs. $4.58 \pm 2.60 \mathrm{~cm}, \mathrm{P}=0.024$; long axis: $2.58 \pm 1.17$ vs. $3.60 \pm$ $2.33 \mathrm{~cm}, \mathrm{P}=0.039)$. The other demographic characteristics are comparable between groups and are summarised in Table S1.

After employing the PSM procedure, there were 69 marched cases including 23 in LTG and 46 in OTG, respectively (Figure 1). The LTG and OTG groups were 


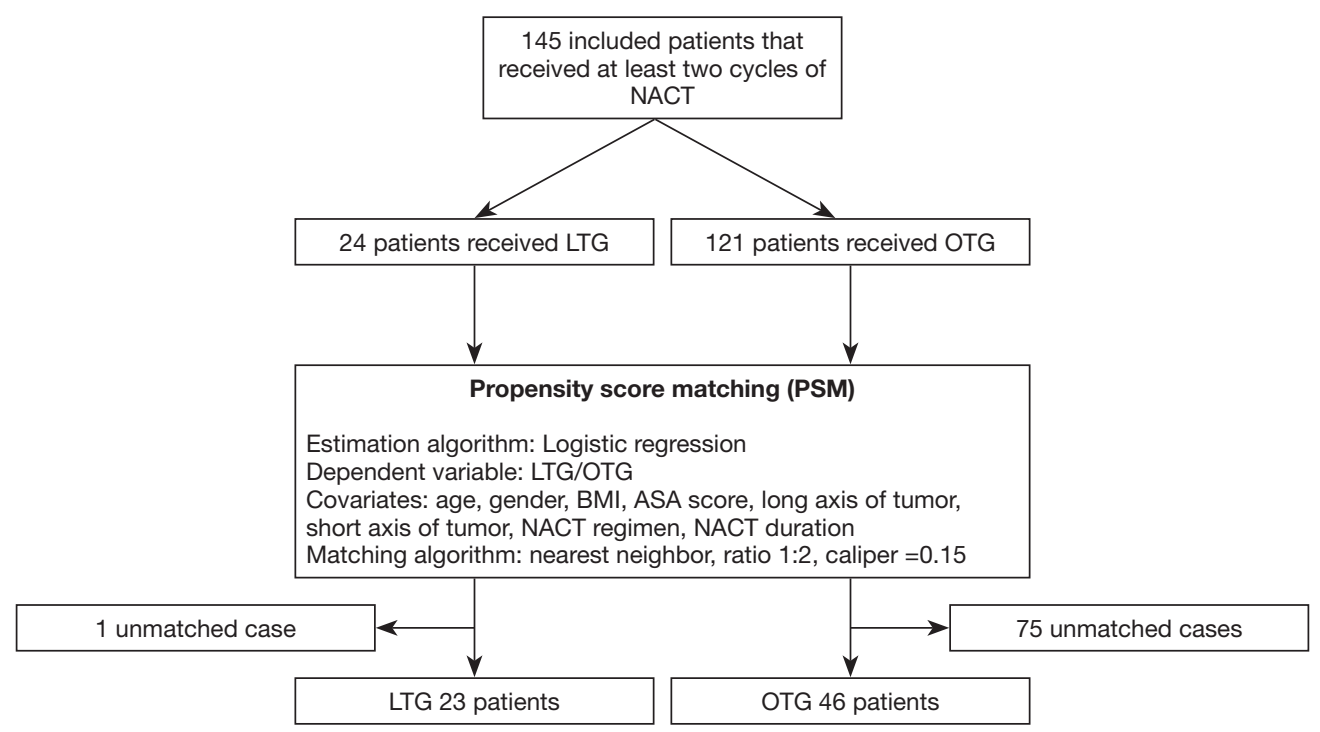

Figure 1 Flowchart showing patient enrolment and PSM matching process. PSM, propensity score matching.
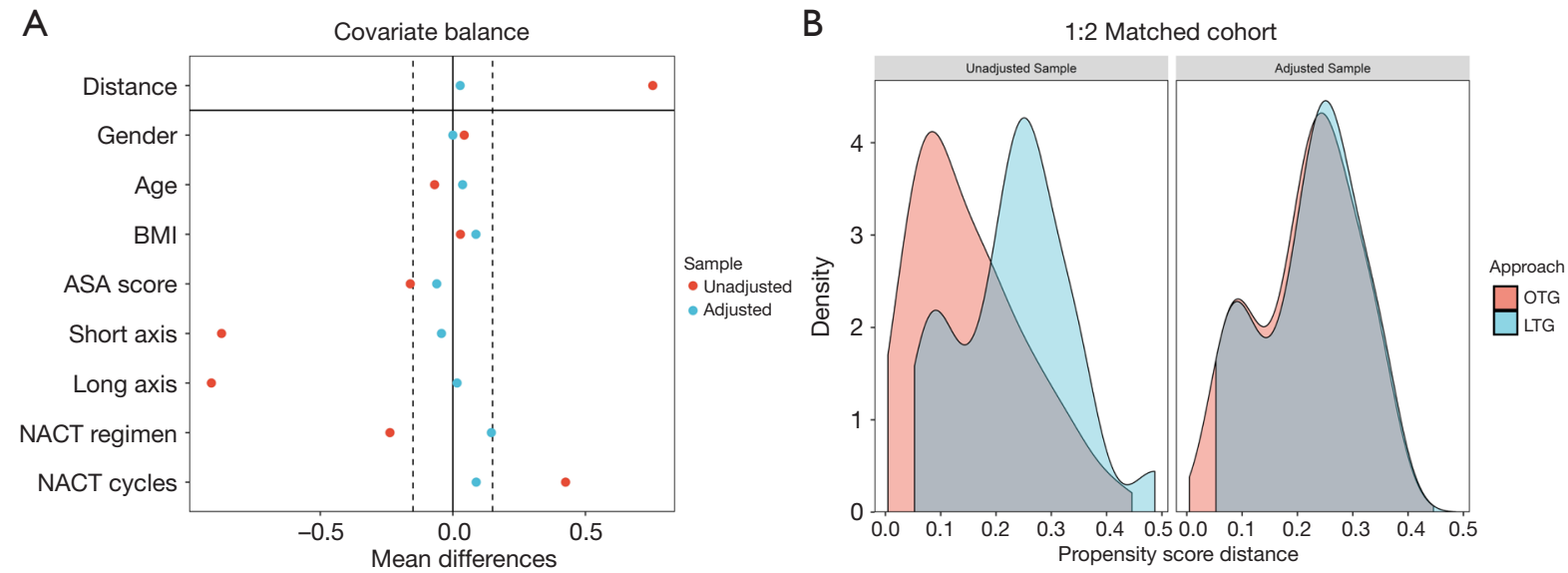

Figure 2 The pre-matching and post-matching information are presented: (A) Love plot demonstrating the value of standardized difference for each covariate included in the propensity score before and after matching. The value of azure dots (after matching) did not exceed the absolute value of 0.15 (shown as dashed line), suggesting a well-balanced distribution for all of the covariates after matching. (B) Propensity score distribution in the LTG and OTG groups before PSM application and after matching. LTG, laparoscopic total gastrectomy; OTG, open total gastrectomy; PSM, propensity score matching.

comparable in terms of age, BMI, gender, ASA classification, comorbidities, and tumour stages and types (Table 1). Area under curve (AUC) for propensity score model was 0.531 . The response rate was $21.74 \%$ and the disease control rate was $97.10 \%$. Response to NACT was not significantly different between the OTG and LTG groups ( $\mathrm{P}=0.633)$.

\section{The radicalness of surgery}

All patients underwent R0 resection. No LTG patients were converted to OTG during surgery. The tumour margin did not differ between the LTG and OTG groups both in proximal (2.5 vs. $2.5 \mathrm{~cm}, \mathrm{P}=1.000)$ and distal (10.0 vs. 
Table 1 Demographic and clinicopathologic characteristics in LTG and OTG group after propensity score matching

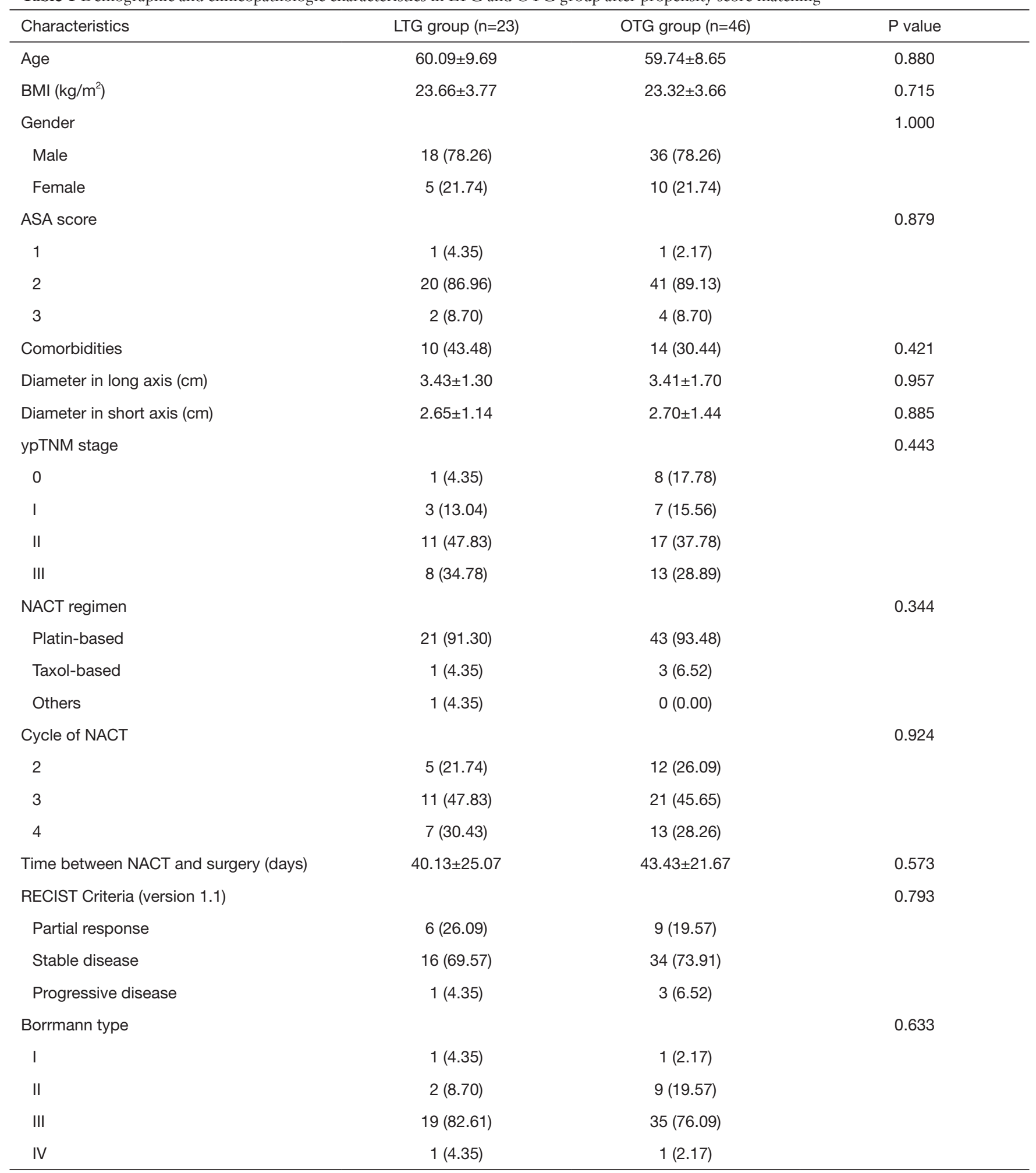

The data are presented as mean \pm SD or $\mathrm{n}(\%)$. BMI, body mass index; ASA, American Society of Anaesthesiologists; NACT, neoadjuvant chemotherapy; RECIST, Response Evaluation Criteria in Solid Tumours; LTG, laparoscopic total gastrectomy; OTG, open total gastrectomy. 
Table 2 Comparison of oncological evaluation of radical surgery between LTG and OTG groups

\begin{tabular}{lccc}
\hline Tumor parameters & LTG group $(\mathrm{n}=23)$ & OTG group $(\mathrm{n}=46)$ & P value \\
\hline Distal margin $(\mathrm{cm})$ & $10.0(8.0,12.0)$ & $10.0(6.5,12.0)$ & 0.692 \\
Proximal margin $(\mathrm{cm})$ & $2.5(1.5,5.0)$ & $2.5(1.5,5.0)$ & 1.000 \\
The number of resected LNs & $37.0(23.0,42.0)$ & $32.0(25.0,38.0)$ & 0.356 \\
The number of metastatic LNs & $1.0(0.0,2.0)$ & $0.0(0.0,3.0)$ & 0.469 \\
Resected LNs of No. 1-6 & $25.0(10.0,34.0)$ & $22.5(16.0,30.0)$ & 0.914 \\
Metastatic LNs of No. 1-6 & $0.0(0.0,1.0)$ & $1.0(0.0,2.0)$ & 0.638 \\
Resected LNs of No. 7-9 & $8.0(7.0,11.0)$ & $0.0(5.0,9.0)$ & 0.082 \\
Metastatic LNs of No. 7-9 & $0.0(0.0,0.0)$ & $0.0(0.0,0.0)$ & 0.313 \\
Resected LNs of No. 11p, 12a & $2.0(1.0,3.0)$ & $1.0(0.0,2.0)$ & 0.107 \\
Metastatic LNs of No. 11p, 12a & $0.0(0.0,0.0)$ & $0.0(0.0,0.0)$ & 0.753 \\
\hline
\end{tabular}

Continuous variates were presented as median (IQR) based on Mann-Whitney U test unless otherwise noted. LNs, lymph nodes; LTG, laparoscopic total gastrectomy; OTG, open total gastrectomy.

$10.0 \mathrm{~cm}, \mathrm{P}=0.692)$. The median number of retrieved LNs was $37.0 \mathrm{LN}$ per patient in the LTG group and 32.0 in the OTG group, with no statistically significant difference between the groups $(\mathrm{P}=0.356)$ (Table 2$)$. In $\mathrm{LN}$ dissection at stations No. 7, 8, and 9, the harvested LNs were slightly higher in LTG comparing to OTG with borderline significance ( 8.0 vs. 7.0, $\mathrm{P}=0.082)$.

\section{Perioperative conditions and recovery}

Perioperative outcomes are shown in Table 3. Compared to the OTG group, the LTG group had a longer average surgery time (262 vs. 205 minutes, $\mathrm{P}<0.001)$ and smaller incision length ( 4.0 vs. $22.0 \mathrm{~cm}, \mathrm{P}<0.001)$. Other measures, including blood loss, length of stay, time to first aerofluxus, defecation, pulling of drainage, and liquid diet, were statistically comparable between LTG and OTG groups, showed no statistically significant difference between the two groups $(\mathrm{P}>0.05)$. None of the surgical cases had intraoperative or postoperative mortality.

Rates of complications were both $34.78 \%$ in LTG $(\mathrm{n}=8)$ and OTG $(\mathrm{n}=16)$ groups, respectively. There was no statistically significant difference between the groups $(\mathrm{P}=1.000)$, nor between the subgroups based on Clavien-Dindo grade $(\mathrm{P}=0.418)$. The occurrence rates of anastomotic leak were $13.04 \%$ in LTG and 8.70 in OTG $(\mathrm{P}=0.573)$. There were five patients experienced III or IV grade of complications: (I) two patients were for toxic shock syndrome (TSS) with respiratory failure that required surgical intervention and management in intensive care unit (ICU). One was for anastomotic leakage that leads to severe peritonitis. The other was secondary to abdominal abscess without anastomotic involvement. All happened in LTG group. (II) Three patients experienced moderate-to-severe systemic signs of systemic infection including high-grade fever and white blood cell count increased over $15.0 \times 10^{9} / \mathrm{L}$ that had evidence of primary infection in the lungs. The symptoms were relieved after thoracentesis and escalating the antibiotic prescriptions. The incidence of different types of complications are shown in Table 3.

All patients received IV-PCA after surgery. The time of use of IV-PCA was significantly shorter in the LTG group than in the OTG group (58.0 vs. 62.0 hours, $\mathrm{P}=0.027$ ) (Table 4). No significant difference in mean supplementary morphine consumption during the hospital stay was detected between the groups $(\mathrm{P}=0.305)$. There were also no significant differences in postoperative pain between the two groups at 24 hours $(\mathrm{P}=1.000), 48$ hours $(\mathrm{P}=0.215)$ and 72 hours $(\mathrm{P}=0.272)$ after surgery (Table 5$)$.

\section{Discussion}

GC has become the second most frequently diagnosed cancer and the third leading cause of cancer-related death in China (4). Although the incidence of GC in China has been decreasing due to the prevention of Helicobacter pylori and the growth of the economy, it still accounts for a significant proportion of all cancer cases. Different from 
Table 3 Comparison of perioperative parameters between LTG and OTG groups

\begin{tabular}{|c|c|c|c|}
\hline Postoperative parameters & LTG group ( $\mathrm{n}=23$ ) & OTG group $(n=46)$ & $P$ value \\
\hline No complication & $15(65.22)$ & $31(67.39)$ & 0.418 \\
\hline Clavien-Dindo grade I/II & $5(21.74)$ & $13(28.26)$ & \\
\hline Clavien-Dindo grade III/IV & $3(13.04)$ & $2(4.35)$ & \\
\hline Intra-abdominal infection & $4(17.39)$ & $5(10.87)$ & 0.448 \\
\hline Pulmonary infection & $2(8.70)$ & $5(10.87)$ & 0.778 \\
\hline All-cause infection & $6(26.09)$ & $10(21.74)$ & 0.687 \\
\hline Hemorrhage/transfusion & $2(8.70)$ & $1(2.17)$ & 0.210 \\
\hline Operation time (min) & $262(234,300)$ & $205(173,235)$ & $<0.001$ \\
\hline Estimated blood loss (mL) & $100(75,120)$ & $100(50,100)$ & 0.764 \\
\hline The first aerofluxus time (days) & $4.0(3.0,4.0)$ & $4.0(3.0,5.0)$ & 0.958 \\
\hline The first defecating time (days) & $5.0(4.0,7.0)$ & $6.0(4.0,7.0)$ & 0.665 \\
\hline Time to pull drainage (days) & $7.0(6.0,11.0)$ & $8.0(6.0,9.0)$ & 0.653 \\
\hline The first time on liquid diets (days) & $6.0(3.0,7.0)$ & $6.0(4.0,8.0)$ & 0.245 \\
\hline Length of stay (days) & $11.0(10.0,15.0)$ & $11.0(10.0,13.0)$ & 0.928 \\
\hline
\end{tabular}

Continuous variates were presented as median (25, 75 percentile). *, Fisher exact test. LTG, laparoscopic total gastrectomy; OTG, open total gastrectomy.

Table 4 Comparison of intravenous patient-controlled analgesia (IV-PCA) application and cumulative morphine consumption between LTG and OTG groups

\begin{tabular}{lccc}
\hline Analgesia regimen & LTG group $(\mathrm{n}=23)$ & OTG group $(\mathrm{n}=46)$ & P value \\
\hline Using IV-PCA & $23(100 \%)$ & $46(100 \%)$ & 0.027 \\
Time of using IV-PCA $(\mathrm{h})$ & $58.0(47.0,64.0)$ & $62.0(58.0,75.0)$ & 0.305 \\
Supplementary morphine consumption $(\mathrm{mg})$ & $120(0,300)$ & $30(0,210)$ & \\
\hline
\end{tabular}

Data are shown as $\mathrm{n}$ (\%) or median (25, 75 percentile). IV-PCA, intravenous patient-controlled analgesia; LTG, laparoscopic total gastrectomy; OTG, open total gastrectomy.

other Eastern countries such as Japan and the Republic of Korea, the majority of GC patients in China are not at an early stage of disease when diagnosed. AGC is associated with a low radical surgical resection rate, high recurrence, and high mortality. Hence, there is an urgent need to find an effective way to cure these patients.

Surgery has always been the cornerstone of AGC treatment. The morbidity and mortality rates of laparoscopy are comparable to those of open resections both in the short-term and long-term (8-10,25-29) and curative resection (R0) with D2 lymphadenectomy is the recommended standard procedure for AGC $(19,30,31)$. On the other hand, since NACT was first introduced in GC (32), it has been recognised as an effective approach to improve the R0 resection rate and kill subclinical micrometastases in the treatment of locally advanced GC $(15,16,33)$. Despite 
Table 5 Comparison of subjective evaluation of acute pain intensity after surgery between LTG and OTG groups (visual analog scale score)

\begin{tabular}{lcc}
\hline Pain scale & LTG group $(\mathrm{n}=23), \mathrm{n}(\%)$ & OTG group $(\mathrm{n}=46), \mathrm{n}(\%)$ \\
\hline Postoperative pain at 24 $\mathrm{h}$ & $21(91.30)$ & $42(91.30)$ \\
Mild [0-3] & $2(8.70)$ & $4(8.70)$ \\
Moderate [4-6] & $0(0.0)$ & $0(0.0)$ \\
Severe [7-10] & & $4000(86.96)$ \\
Postoperative pain at $48 \mathrm{~h}$ & $17(73.91)$ & $6(13.04)$ \\
Mild [0-3] & $5(21.74)$ & $0(0.00)$ \\
Moderate [4-6] & $1(4.35)$ & $0.215^{*}$ \\
Severe [7-10] & & $38(82.61)$ \\
Postoperative pain at $72 \mathrm{~h}$ & $15(65.22)$ & $6(13.04)$ \\
Mild [0-3] & $6(26.09)$ & $2(4.35)$ \\
Moderate [4-6] & $2(8.70)$ & 0.272 \\
Severe [7-10] & & \\
\hline
\end{tabular}

*, Fisher exact test. LTG, laparoscopic total gastrectomy; OTG, open total gastrectomy.

the variation in NACT regimens adopted at different centres, several high-quality trials with specific NACT arms, such as MAGIC and FNCLCC/FFCD, have already shown a benefit for overall survival. Until now, there has been little available evidence on the short-term benefit of LTG after NACT (34). Many recent studies have focused on the outcomes of LTG and OTG but have not examined the combined role of NACT $(8,9,28,35)$. In our previous study focusing on laparoscopic distal gastrectomy (LDG) following NACT treatment, the results showed comparable surgical outcomes to the open group (ODG) (36). However, this conclusion cannot be directly generalised to LTG patients since LTG appears to be associated with greater postoperative and surgical complications. Further, this more sophisticated technique requires a longer duration of nursing care and hospital stay. There are still concerns about the damage to the intestinal barrier and reduced probiotics due to NACT $(37,38)$. The NACT, on the other hand, might affect the outcomes of the procedure in varying degrees according to the type of resection. Téoule et al. studied 135 patients, among which $90 \%$ of the NACT underwent total gastrectomy, and found that, compared to radical gastrectomy, NACT might increase the rate of wound infection and duodenal stump leakage (39). Thus, the safety and efficacy of the laparoscopic procedure after NACT is still unclear.

In our study, the CapeOX, SOX, and DS regimens accounted for nearly $90 \%$ of all NACT regimens; these are all commonly used for perioperative chemotherapy [e.g., CapeOX in CLASSIC (40), DS in START (41), and SOX in CAPITAL and ARTIST-II study]. The response rate to NACT was $22.1 \%$ among all patients and over half of the SD patients achieved a reduction in tumour or LN size. The disease control rate was $97.2 \%$ and all patients underwent R0 resection with no LTG cases converting to open surgery. These results provide evidence of the shortterm benefits of NACT.

Compared to OTG after NACT, the LTG group had an increased surgery time but a shorter length of incision and did not differ significantly in terms of blood loss, postoperative complications, length of stay, and time on liquid diet after surgery. The complications rate of the two groups did not differ significantly, though the rate in the LTG group was greater than the OTG group by $13.5 \%$, which may be attributed to the relatively low number of LTG patients. The number of harvested LNs is now regarded as an independent prognostic factor in AGC $(42,43)$. All patients in our study underwent D2 dissection. The median number of LNs was 37 in the LTG group, which was slightly higher than that in the OTG group. Although LTG has been, previously, associated with inferior LN retrieval in some studies (44), Chen et al. reported that laparoscopic gastrectomy is more suitable for LNs diameter from 1.0 to $1.9 \mathrm{~cm}$ than open method in an experienced 
surgical team (45). Our findings again suggest that laparoscopic technique does not account for the reduction of LNs retrieval, which warrant the credibility of histological findings $(46,47)$. Though not fully in accordance with our previous results on distal gastrectomy (36), the findings is consistent with the basic premise that patients who undergo LTG have comparable surgical outcomes to open surgery.

There is still debate as to whether NACT increases postoperative morbidity and mortality $(38,48,49)$. In the current study, morbidity rates were $34.78 \%$ both in LTG and OTG groups, including the incidence of infection with $23.19 \%$ for all-cause and $13.04 \%$ for intra-abdomen. Although the morbidity rate were reasonable compared to previous reports in patients without NACT, ranging from $4.8 \%$ to $52.6 \%$ for LTG (50-53), and $3.5-43 \%$ for OTG $(52,54-56)$, it was higher than our previous published results with $24.7 \%$ complications rate for patients undergoing gastrectomy (57). Pointed out by Yuan et al., either laparoscopy, or surgical approach or use of NACT was not the independent predictor for postoperative complications. However, the complications rate in NACT patients was $30.23 \%$ (comparable to our $34.78 \%$ ), a bit higher than non-NACT group (21.57\%) in their study. Thus, whether NACT increases the incidence of postoperative complications, especially for infections, still require larger parallel-group investigations (39).

All patients in our study received postoperative IVPCA and we used the VAS to evaluate pain intensity. The duration of time spent on IV-PCA was significantly shorter in the LTG compared to the OTG group, while the use of supplementary morphine was comparable; there were no statistical differences between two groups in the first 24 to $72 \mathrm{~h}$ after surgery. The shortened duration of IV-PAC in the LTG group indicates a lower dose of postoperative opioids and better pain relief after surgery; this can benefit patients in terms of reducing their risk of PCA-related adverse effects such as nausea, vomiting, headache, dizziness, and sedation (58) and is less likely to mask signs of postoperative complications (59).

There are limitations of this study that should be noted. Though it was designed as a prospective cohort study, the choice of surgical approach was based on each patient's preference and was not subject to randomisation or blinding. There was also an unequal number of patients in each group and the sample size in the LTG group was relatively small. Lastly, this single-centre study focused on the short-term outcomes of LTG and OTG after NACT. This study lacks long-term follow-up, so we cannot draw any conclusions about survival benefits.

\section{Conclusions}

In conclusion, this study showed that LTG after NACT had comparable perioperative safety and histological findings to OTG after NACT. However, LTG is less invasive and was associated with less IV-PCA use after surgery. Further research is needed to investigate the short-term complications and long-term effects of LTG after NACT in the treatment of AGC.

\section{Acknowledgments}

Funding: This work was funded by Beijing Municipal Health Commission (DFL20181103, ZYLX201701).

\section{Footnote}

Reporting Checklist: The authors have completed the STROBE reporting checklist. Available at http://dx.doi. org/10.21037/jgo-20-374

Data Sharing Statement: Available at http://dx.doi. org/10.21037/jgo-20-374

Peer Review File: Available at http://dx.doi.org/10.21037/ jgo-20-374

Conflicts of Interest: All authors have completed the ICMJE uniform disclosure form (available at http://dx.doi. org/10.21037/jgo-20-374). The authors have no conflicts of interest to declare.

Ethical Statement: The authors are accountable for all aspects of the work in ensuring that questions related to the accuracy or integrity of any part of the work are appropriately investigated and resolved. The study was conducted in accordance with the Declaration of Helsinki (as revised in 2013). The study was approved by Ethics Committee of Beijing Cancer Hospital (No. 2019YJZ26) and informed consent was taken from all the patients.

Open Access Statement: This is an Open Access article distributed in accordance with the Creative Commons Attribution-NonCommercial-NoDerivs 4.0 International License (CC BY-NC-ND 4.0), which permits the noncommercial replication and distribution of the article with 
the strict proviso that no changes or edits are made and the original work is properly cited (including links to both the formal publication through the relevant DOI and the license). See: https://creativecommons.org/licenses/by-nc-nd/4.0/.

\section{References}

1. Bray F, Ferlay J, Soerjomataram I, et al. Global cancer statistics 2018: GLOBOCAN estimates of incidence and mortality worldwide for 36 cancers in 185 countries. CA Cancer J Clin 2018;68:394-424.

2. Yang L, Zheng R, Wang N, et al. Incidence and mortality of stomach cancer in China, 2014. Chinese journal of cancer research $=$ Chung-kuo yen cheng yen chiu 2018;30:291-8.

3. Zong L, Abe M, Seto Y, et al. The challenge of screening for early gastric cancer in China. Lancet 2016;388:2606.

4. Feng RM, Zong YN, Cao SM, et al. Current cancer situation in China: good or bad news from the 2018 Global Cancer Statistics? Cancer Commun (Lond) 2019;39:22.

5. Rawla P, Barsouk A. Epidemiology of gastric cancer: global trends, risk factors and prevention. Prz Gastroenterol 2019;14:26-38.

6. Azagra JS, Goergen M, Ansay J, et al. Laparoscopic gastric reduction surgery. Preliminary results of a randomized, prospective trial of laparoscopic vs open vertical banded gastroplasty. Surg Endosc 1999;13:555-8.

7. Jeong AR, Park JH, Yang JY, et al. A comparison of shortterm outcomes of laparoscopic-assisted total gastrectomy and open total gastrectomy in early gastric cancer. J Clin Oncol 2017;13:55-61.

8. Kawamura H, Yokota R, Homma S, et al. Comparison of invasiveness between laparoscopy-assisted total gastrectomy and open total gastrectomy. World J Surg 2009;33:2389.

9. Hong L, Han Y, Jin Y, et al. The short-term outcome in esophagogastric junctional adenocarcinoma patients receiving total gastrectomy: Laparoscopic versus open gastrectomy - A retrospective cohort study. Int J Surg 2013;11:957-61.

10. Chen K, Pan Y, Zhai ST, et al. Totally laparoscopic versus open total gastrectomy for gastric cancer: A casematched study about short-term outcomes. Medicine 2017;96:e8061.

11. Pacelli F, Cusumano G, Rosa F, et al. Multivisceral Resection for Locally Advanced Gastric Cancer: An Italian Multicenter Observational StudyMultivisceral Resection for Gastric Cancer. JAMA Surg 2013;148:353-60.
12. Coburn NG. Lymph nodes and gastric cancer. J Surg Oncol 2009;99:199-206.

13. Jiang L, Yang KH, Guan QL, et al. Survival benefit of neoadjuvant chemotherapy for resectable cancer of the gastric and gastroesophageal junction. J Clin Gastroenterol 2015;49:387-94.

14. Biffi R, Fazio N, Luca F, et al. Surgical outcome after docetaxel-based neoadjuvant chemotherapy in locallyadvanced gastric cancer. World J Gastroenterol 2010;16:868.

15. Schuhmacher C, Gretschel S, Lordick F, et al. Neoadjuvant chemotherapy compared with surgery alone for locally advanced cancer of the stomach and cardia: European Organisation for Research and Treatment of Cancer randomized trial 40954. J Clin Oncol 2010;28:5210.

16. Cunningham D, Allum WH, Stenning SP, et al. Perioperative chemotherapy versus surgery alone for resectable gastroesophageal cancer. $\mathrm{N}$ Engl J Med 2006;355:11-20.

17. In H, Ravetch E, Langdon-Embry M, et al. The newly proposed clinical and post-neoadjuvant treatment staging classifications for gastric adenocarcinoma for the American Joint Committee on Cancer (AJCC) staging. Gastric Cancer 2018;21:1-9.

18. Ajani JA, D'Amico TA, Almhanna K, et al. Gastric cancer, version 3.2016, NCCN clinical practice guidelines in oncology. J Natl Compr Canc Netw 2016;14:1286-312.

19. Japanese Gastric Cancer Association. Japanese gastric cancer treatment guidelines 2014 (ver. 4). Gastric Cancer 2017;20:1-19.

20. Eisenhauer EA, Therasse P, Bogaerts J, et al. New response evaluation criteria in solid tumours: revised RECIST guideline (version 1.1). Eur J Cancer 2009;45:228-47.

21. Downie WW, Leatham P, Rhind V, et al. Studies with pain rating scales. Ann Rheum Dis 1978;37:378-81.

22. Centers for Medicare and Medicaid Services. Opioid Oral Morphine Milligram Equivalent (MME) Conversion Factors. Available online: https://www. cms.gov/Medicare/Prescription-Drug-Coverage/ PrescriptionDrugCovContra/Downloads/OpioidMorphine-EQ-Conversion-Factors-Aug-2017.pdf

23. Clavien PA, Sanabria JR, Strasberg SM. Proposed classification of complications of surgery with examples of utility in cholecystectomy. Surgery 1992;111:518-26.

24. Ho DE, Imai K, King G, et al. MatchIt: Nonparametric Preprocessing for Parametric Causal Inference. Journal of Statistical Software 2011. doi: 10.18637/jss.v042.i08.

25. Cuschieri A, Fayers P, Fielding J, et al. Postoperative 
morbidity and mortality after D1 and D2 resections for gastric cancer: preliminary results of the MRC randomised controlled surgical trial. The Surgical Cooperative Group. Lancet 1996;347:995-9.

26. Yu J, Huang C, Sun Y, et al. Effect of Laparoscopic vs Open Distal Gastrectomy on 3-Year Disease-Free Survival in Patients With Locally Advanced Gastric Cancer: The CLASS-01 Randomized Clinical Trial. JAMA 2019;321:1983-92.

27. Degiuli M, Sasako M, Ponti A. Morbidity and mortality in the Italian Gastric Cancer Study Group randomized clinical trial of D1 versus D2 resection for gastric cancer. Br J Surg 2010;97:643-9.

28. Kim MG, Kim BS, Kim TH, et al. The effects of laparoscopic assisted total gastrectomy on surgical outcomes in the treatment of gastric cancer. J Korean Surg Soc 2011;80:245-50.

29. Sheng S, Chen Y, Li C. Outcomes of Laparoscopic Total Gastrectomy for Elderly Gastric Cancer Patients. J Cancer 2018;9:4398.

30. Lee JH, Kim JG, Jung HK, et al. Clinical practice guidelines for gastric cancer in Korea: an evidence-based approach. J Gastric Cancer 2014;14:87-104.

31. Smyth EC, Verheij M, Allum W, et al. Gastric cancer: ESMO Clinical Practice Guidelines for diagnosis, treatment and follow-up. Ann Oncol 2016;27:v38-v49.

32. Stephens FO, Harker GJ, Dickinson RT, et al. Preoperative basal chemotherapy in the management of cancer of the stomach: a preliminary report. Aust N Z J Surg 1979;49:331-5.

33. Ychou M, Boige V, Pignon JP, et al. Perioperative chemotherapy compared with surgery alone for resectable gastroesophageal adenocarcinoma: an FNCLCC and FFCD multicenter phase III trial. J Clin Oncol 2011;29:1715-21.

34. Fujisaki M, Mitsumori N, Shinohara T, et al. Shortand long-term outcomes of laparoscopic versus open gastrectomy for locally advanced gastric cancer following neoadjuvant chemotherapy. Surg Endosc 2021;35:1682-90.

35. Shim JH, Oh SI, Yoo HM, et al. Short-term outcomes of laparoscopic versus open total gastrectomy: a matchedcohort study. Am J Surg 2013;206:346-51.

36. Li Z, Shan F, Wang Y, et al. Laparoscopic versus open distal gastrectomy for locally advanced gastric cancer after neoadjuvant chemotherapy: safety and short-term oncologic results. Surg Endosc 2016;30:4265-71.

37. Li Z, Shan F, Ying X, et al. Assessment of Laparoscopic Distal Gastrectomy After Neoadjuvant Chemotherapy for
Locally Advanced Gastric Cancer: A Randomized Clinical Trial. JAMA Surg 2019;154:1093-101.

38. Wei Z, Tan B, Cao S, et al. The influence of neoadjuvant chemotherapy on gastric cancer patients' postoperative infectious complications: What is the negative role played by the intestinal barrier dysfunction? Oncotarget 2017;8:43376.

39. Téoule P, Trojan J, Bechstein W, et al. Impact of neoadjuvant chemotherapy on postoperative morbidity after gastrectomy for gastric cancer. Dig Surg 2015;32:229-37.

40. Bang YJ, Kim YW, Yang HK, et al. Adjuvant capecitabine and oxaliplatin for gastric cancer after D2 gastrectomy (CLASSIC): a phase 3 open-label, randomised controlled trial. Lancet 2012;379:315-21.

41. Koizumi W, Kim YH, Fujii M, et al. Addition of docetaxel to $\mathrm{S}-1$ without platinum prolongs survival of patients with advanced gastric cancer: a randomized study (START). J Cancer Res Clin Oncol 2014;140:319-28.

42. Shi RL, Chen Q, Ding JB, et al. Increased number of negative lymph nodes is associated with improved survival outcome in node positive gastric cancer following radical gastrectomy. Oncotarget 2016;7:35084.

43. Kinoshita O, Ichikawa D, Ichijo Y, et al. Histological evaluation for chemotherapeutic responses of metastatic lymph nodes in gastric cancer. World J Gastroenterol 2015;21:13500-6.

44. Xiong JJ, Nunes QM, Huang W, et al. Laparoscopic vs open total gastrectomy for gastric cancer: a meta-analysis. World J Gastroenterol 2013;19:8114-32.

45. Chen QY, Zheng CH, Li P, et al. Which method is more suitable for advanced gastric cancer with enlarged lymph nodes, laparoscopic radical gastrectomy or open gastrectomy? Gastric Cancer 2018;21:853-63.

46. Smith DD, Schwarz RR, Schwarz RE. Impact of total lymph node count on staging and survival after gastrectomy for gastric cancer: data from a large USpopulation database. J Clin Oncol 2005;23:7114-24.

47. Strong VE, Song KY, Park CH, et al. Comparison of gastric cancer survival following R0 resection in the United States and Korea using an internationally validated nomogram. Ann Surg 2010;251:640-6.

48. Grabenbauer GG, Holger G. Management of radiation and chemotherapy related acute toxicity in gastrointestinal cancer. Best Pract Res Clin Gastroenterol 2016;30:655-64.

49. Li ZY, Shan F, Zhang LH, et al. Complications after radical gastrectomy following FOLFOX7 neoadjuvant chemotherapy for gastric cancer. World J Surg Oncol 
2011;9:110.

50. Jeong O, Jung MR, Kim GY, et al. Comparison of shortterm surgical outcomes between laparoscopic and open total gastrectomy for gastric carcinoma: case-control study using propensity score matching method. J Am Coll Surg 2013;216:184-91.

51. Shinohara T, Kanaya S, Taniguchi K, et al. Laparoscopic total gastrectomy with D2 lymph node dissection for gastric cancer. Arch Surg 2009;144:1138-42.

52. Hu Y, Huang C, Sun Y, et al. Morbidity and mortality of laparoscopic versus open D2 distal gastrectomy for advanced gastric cancer: a randomized controlled trial. J Clin Oncol 2016;34:1350-7.

53. Lee JH, Ahn SH, Park DJ, et al. Laparoscopic total gastrectomy with D2 lymphadenectomy for advanced gastric cancer. World J Surg 2012;36:2394-9.

54. Degiuli M, Sasako M, Calgaro M, et al. Morbidity and mortality after D1 and D2 gastrectomy for cancer: interim analysis of the Italian Gastric Cancer Study Group (IGCSG) randomised surgical trial. Eur J Surg Oncol

Cite this article as: Wang Y, Lei X, Liu Z, Shan F, Ying X, Li Z, Ji J. Short-term outcomes of laparoscopic versus open total gastrectomy after neoadjuvant chemotherapy: a cohort study using the propensity score matching method. J Gastrointest Oncol 2021;12(2):237-248. doi: 10.21037/jgo-20-374
2004;30:303-8.

55. Bonenkamp JJ, Songun I, Welvaart K, et al. Randomised comparison of morbidity after D1 and D2 dissection for gastric cancer in 996 Dutch patients. Lancet 1995;345:745-8.

56. Kim HH, Hyung WJ, Cho GS, et al. Morbidity and mortality of laparoscopic gastrectomy versus open gastrectomy for gastric cancer: an interim report-a phase III multicenter, prospective, randomized Trial (KLASS Trial). Ann Surg 2010;251:417-20.

57. Yuan $\mathrm{P}, \mathrm{Wu} Z$, Li Z, et al. Impact of postoperative major complications on long-term survival after radical resection of gastric cancer. BMC Cancer 2019;19:833.

58. Koh JC, Lee J, Kim SY, et al. Postoperative Pain and Intravenous Patient-Controlled Analgesia-Related Adverse Effects in Young and Elderly Patients: A Retrospective Analysis of 10,575 Patients. Medicine 2015;94:e2008.

59. Macintyre PE. Safety and efficacy of patient-controlled analgesia. Br J Anaesth 2001;87:36-46. 


\section{Supplementary}

Table S1 Dosage and schedule of the treatment regimen

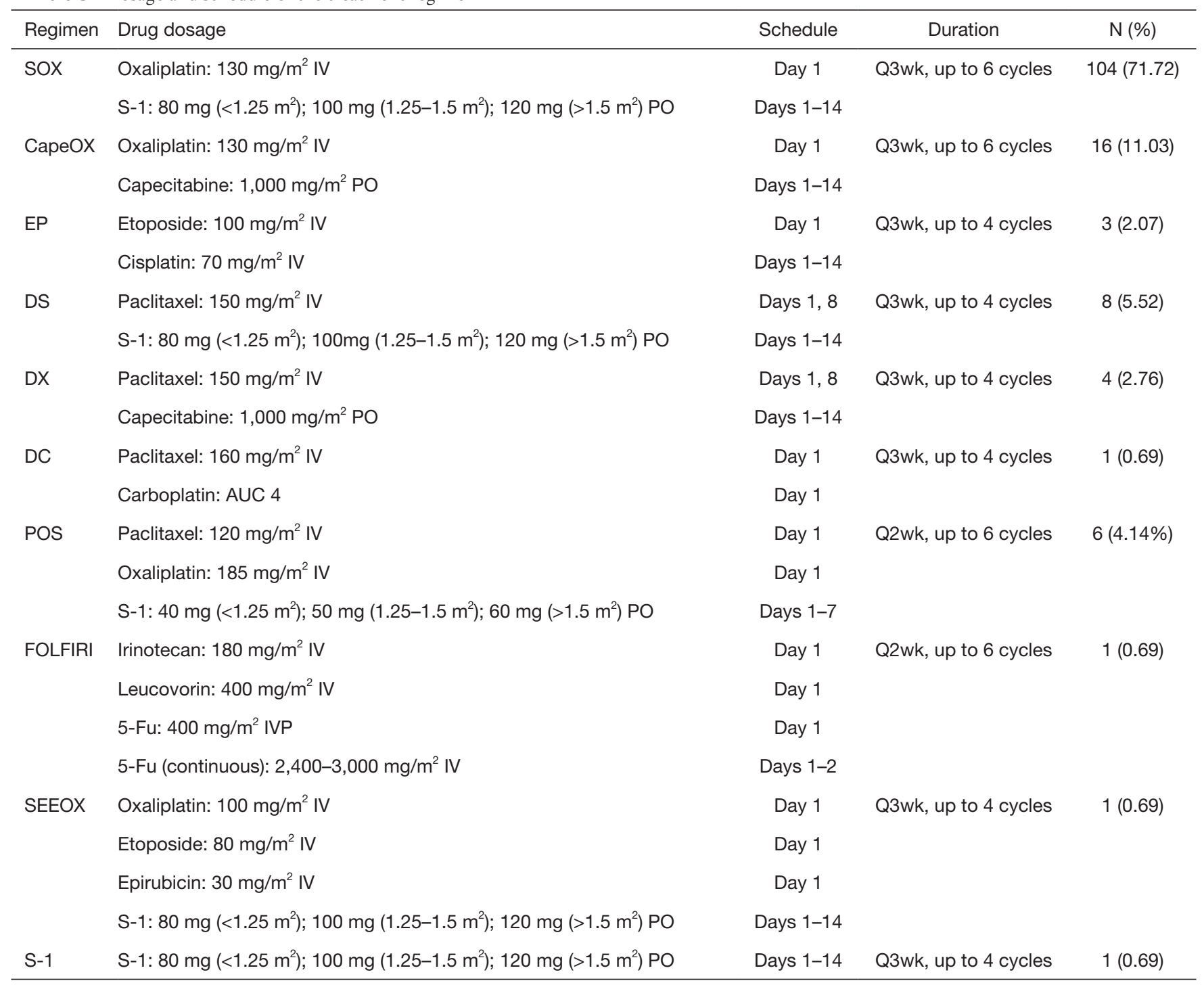

$\mathrm{PO}$, by oral; IV, intravenous. 
Table S2 Demographic and clinicopathologic characteristics in LTG and OTG group before propensity score matching

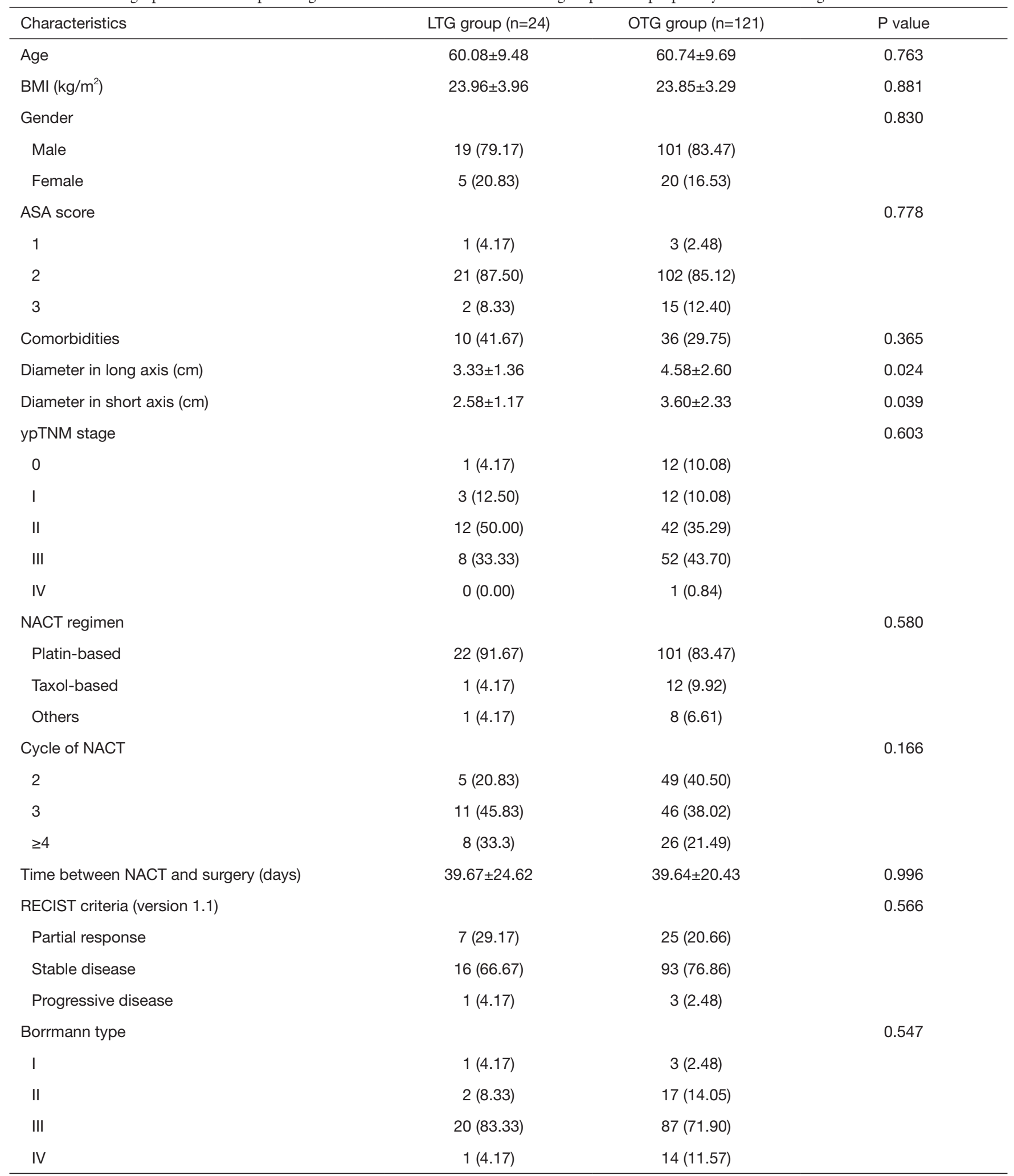

BMI, body mass index; ASA, American Society of Anaesthesiologists; NACT, neoadjuvant chemotherapy; RECIST, Response Evaluation Criteria in Solid Tumours; LTG, laparoscopic total gastrectomy; OTG, open total gastrectomy. 